\title{
AVALIAÇÃO DO POTENCIAL ENZIMÁTICO DE ENZIMAS COMERCIAIS NA HIDRÓLISE DE BAGAÇO DE CANA-DE- AÇÚCAR DO RIO GRANDE DO NORTE
}

\author{
L. P. DE SOUZA ${ }^{1}$, P. V. F. DANTAS ${ }^{1}$, A. DE A. GUILHERME ${ }^{1}$, E. S. DOS SANTOS ${ }^{1}$, F. A. \\ N. FERNANDES ${ }^{2}$, G. R. DE MACEDO ${ }^{1}$ \\ ${ }^{1}$ Universidade Federal do Rio Grande do Norte - Departamento de Engenharia Química \\ ${ }^{2}$ Universidade Federal do Ceará - Departamento de Engenharia Química \\ Contato: alexandredearaujoguilherme@ gmail.com
}

\begin{abstract}
RESUMO: devido a questões econômicas e ambientais, a substituição da matriz energética do petróleo pela biomassa vegetal, torna-se importante no cenário mundial, visto que é uma fonte renovável de energia. Este trabalho teve como objetivo a comparação de diferentes enzimas para hidrólise enzimática do bagaço de cana pré-tratado físico-quimicamente. $\mathrm{O}$ bagaço foi pré-tratado com ácido sulfúrico em uma primeira etapa e hidróxido de sódio em uma segunda etapa e em seguida foi hidrolisado enzimaticamente. Os ensaios de hidrólise enzimática foram realizados em incubador rotatório em erlenmyers de $250 \mathrm{~mL}$ com $20 \mathrm{~mL}$ de volume reacional. As enzimas estudadas foram celulases, xilanases, hemicelulases e $\beta$ glicosidases, todas cedidas por empresas multinacionais. A partir dos resultados encontrados, foi possível obter até $60 \%$ de conversão de celulose em glicose e obter resultados para estudos econômicos em termos de aplicação das enzimas xilanases e hemicelulases.
\end{abstract}

\section{INTRODUÇÃO}

A biomassa vegetal rica em material lignocelulósico está sendo estudado no mundo todo como uma fonte renovável de carbono e energia. Sem dúvida alguma, o etanol é o mais importante produto final obtido pela fermentação de hidrolisados lignocelulósicos, porém outros produtos também podem ser obtidos.

Para a produção de etanol de segunda geração (etanol celulósico) é preciso que se obtenham monômeros de carboidratos como a glicose proveniente da estrutura da parede celular vegetal, lignocelulose. Desta forma, diferentemente do processo convencional, para a produção de etanol de segunda geração é preciso que o material lignocelulósico seja submetido a um pré-tratamento químico, físico, biológico ou combinado e a uma hidrólise enzimática para que se tenha substrato disponível para a fermentação alcoólica.

Na hidrólise enzimática da celulose são necessário pelo menos três tipos de enzimas para se que se obtenha glicose. As endoglicanases (EG) (EC.3.2.1.4) hidrolisam as moléculas de celulose de forma randômica produzindo oligômeros. As celobiohidrolases ou exoglicanases $(\mathrm{CBH})$ (EC.3.2.1.91) convertem os oligômeros, formados pela ação das 


\section{9 a 22 de outubro de 2014 \\ Florianópolis/SC}

endoglicanases, em celobiose e celodextrinas solúveis. A $\beta$-glicosidase (BG), também chamada de celobiase, (EC.3.2.1.21) converte celobiose e celodextrinas em glicose (Zhou et al., 2009).

As enzimas celobiohidrolases agem nas extremidades dos oligômeros sendo então de dois tipos, uma que age na extremidade redutora e o outro tipo que age na extremidade nãoredutora (Lynd et al., 2002). Todas estas enzimas trabalham sinergicamente para hidrolisar a celulose (Galbe e Zacchi, 2002).

As enzimas endo e exoglicanases são denominadas de celulases e são geralmente produzidas por linhagens de Trichoderma sp. (Zhou et al., 2009). Para uma maior eficiência no processo industrial de hidrólise de celulose à glicose, as enzimas celulases precisam ser suplementadas com a $\beta$-glicosidase e esta geralmente é produzida por linhagens de Aspergillus niger (Han e Chen, 2008).

Este trabalho teve como objetivo estudar o potencial enzimático de enzimas comerciais na hidrólise enzimática de bagaço de cana-de-açúcar pré-tratado visando à produção de glicose para futura fermentação alcóolica.

\section{MATERIAL E MÉTODOS}

\subsection{Enzimas}

As enzimas utilizadas neste trabalho foram as celulases (Celluclast 1.5L), a enzima $\beta$ glicosidase (Novozym 188), o complexo de celulases (NS22074), a enzima $\beta$-glicosidase (NS50010), o complexo enzimático com arabinase, $\beta$-glicanase, celulases, hemicelulases, pectinase e xilanase (NS50012) e enzima xilanase (NS22036), todas cedidas pela Novozymes (Bagsvaerd, Dinamarca) e o complexo enzimático (Accellerase 1500) cedida pela (Danisco/GENENCOR, Dinamarca). As atividades enzimáticas das enzimas foram determinadas segundo Ghose (1987) para caracterização e composição das misturas enzimáticas testadas.

\subsection{Bagaço de cana-de-açúcar e pré-tratamento}

O substrato utilizado neste trabalho foi o bagaço de cana-de-açúcar cedido gentilmente pela Usina Estivas (Arês - RN, Brasil) e submetido a um pré-tratamento com ácido sulfúrico diluído em uma primeira etapa e hidróxido de sódio em uma segunda etapa.

Para o pré-tratamento foi utilizado bagaço na quantidade de sólidos de $20 \%$ (p/v) imerso em uma solução de ácido sulfúrico $2 \%$ (v/v) submetido a uma temperatura de $121^{\circ} \mathrm{C}$ por 30 minutos, segundo Guo et al. (2009). Em seguida, a fração sólida foi lavada com água até o valor do $\mathrm{pH}$ ficar próximo ao $\mathrm{pH}$ da água de lavagem, e seca a $40^{\circ} \mathrm{C}$ em estufa com circulação e renovação de ar por $24 \mathrm{~h}$. Esta primeira etapa é para remoção, principalmente, da hemicelulose. 
Após o pré-tratamento ácido, o material foi submetido a um pré-tratamento alcalino para a deslignificação onde foi utilizada uma quantidade de $20 \%(\mathrm{p} / \mathrm{v})$ do material sólido submerso em solução de hidróxido de sódio $4 \%$ (p/v) submetido a uma temperatura de $121^{\circ} \mathrm{C}$ por 30 minutos, segundo Vasquez et al. (2007). Em seguida, o pH foi corrigido para 7,0 utilizando ácido clorídrico para posterior lavagem. Após a lavagem, o material foi seco a $40^{\circ} \mathrm{C}$ em estufa com circulação e renovação de ar por $24 \mathrm{~h}$ para estocagem.

O bagaço utilizado neste estudo tinha a granulometria com dimensões iguais e inferiores a $0,29 \mathrm{~mm}$ de diâmetro. Para isto, a biomassa foi triturada e peneirada (50 mesh) antes de ser submetido ao pré-tratamento.

\subsection{Composição do bagaço de cana}

O bagaço de cana in natura e pré-tratado foi caracterizado quanto às quantidades de celulose, hemicelulose, lignina total, cinzas, extrativos em solvente orgânicos e extrativos em água quente $\left(100{ }^{\circ} \mathrm{C}\right)$. As quantificações de celulose, hemicelulose, lignina e cinzas foram realizadas de acordo com Gouveia et al., (2009). As determinações dos extrativos em solvente orgânico e em água quente foram realizadas segundo o NREL (National Renewable Energy Laboratory, Golden, Colorado - USA), conforme Sluiter et al., 2008.

\subsection{Ensaios enzimáticos}

Na Tabela 1 estão demonstrados os ensaios realizados para seleção da mistura enzimática, bem como, outros testes, visando um aumento na produção de glicose.

Tabela 1 - Ensaios variando diferentes misturas enzimáticas e TWEEN 80.

\begin{tabular}{ll}
\multicolumn{1}{c}{ Ensaios } & \multicolumn{1}{c}{ Condições testadas } \\
\hline Primeiro estudo & (Celluclast 1.5L) + (Novozym 188) \\
Segundo estudo & (Accellerase 1500) \\
Terceiro estudo & (NS22074) + (NS50010) \\
Quarto estudo & Primeiro estudo + (NS22036) $(0,25 \%)(\mathrm{v} / \mathrm{v})$ \\
Quinto estudo & Primeiro estudo + (NS50012) $(1 \%)(\mathrm{v} / \mathrm{v})$ \\
Sexto estudo & Primeiro estudo $+(\mathrm{NS} 22036)(0,25 \%)(\mathrm{v} / \mathrm{v})+$ TWEEN $80(0,05 \mathrm{~g} / \mathrm{g}$ bagaço $)$ \\
\hline
\end{tabular}

Os experimentos de hidrólise enzimática no bagaço de cana pré-tratado foram realizados em ensaios independentes de 5, 10, 24 e 48 horas em triplicata. As hidrólises ocorreram em $20 \mathrm{ml}$ de volume reacional com 1,4\% de bagaço em erlenmeyers de $250 \mathrm{ml}$ o que corresponde a $1 \%$ de celulose, os quais foram colocados em incubador rotatório a $50{ }^{\circ} \mathrm{C} \mathrm{e}$ $150 \mathrm{rpm}$ de acordo com o protocolo de digestibilidade da NREL (Selig et al., 2008). As enzimas foram adicionadas com as atividades iniciais de $60 \mathrm{FPU} / \mathrm{g}$ celulose para as celulases e $30 \mathrm{CBU} / \mathrm{g}$ celulose para as $\beta$-glicosidases.

Para este estudo, o bagaço de cana pré-tratado não foi lavado com água quente (100 $\left.{ }^{\circ} \mathrm{C}\right)$ para ser usado nas hidrólises enzimáticas, portanto, os açúcares impregnados da matriz 
lignocelulosica (denominados de extraíveis em água quente) foram incorporados aos resultados de produção de glicose.

A quantidade de celulose digerida (conversão de celulose em glicose) foi determinada de forma indireta segundo o protocolo de avaliação de digestibilidade da biomassa lignocelulósica do NREL (Selig et al., 2008), e expressa conforme Equações 1 e 2:

$$
(\%) \text { Conversão }=\frac{\text { celulose digerida }(g)}{\text { celulose adicionada }(g)} \times 100
$$

$$
\text { celulose digerida }(g)=0.9 \times(\text { Volume reacional }(L)) \times \operatorname{gli} \cos e(g / L)
$$

Os resultados de conversão foram analisados através do teste de diferença entre as médias $($ Teste $-\mathrm{t})$ com $(\alpha=0.5)$ realizado no software Statistica v7 para se comparar o desempenho das enzimas estudadas em cada tempo de ensaio.

\section{RESULTADOS E DISCUSSÃO}

Na Tabela 2 estão apresentados os resultados da caracterização das enzimas aplicadas neste trabalho.

Tabela 2 - resultados da caracterização das enzimas.

\begin{tabular}{lccc}
\hline \multicolumn{1}{c}{ Enzimas } & $\begin{array}{c}\text { Atividade celulase } \\
(\mathbf{F P U} / \mathbf{m L})\end{array}$ & $\begin{array}{c}\text { Atividade } \boldsymbol{\beta} \text {-glicosidase } \\
(\mathbf{C B U} / \mathbf{m L})\end{array}$ & $\begin{array}{c}\text { Atividade xilanase } \\
(\text { FXU/ml) }\end{array}$ \\
\hline Celluclast 1.5 & $109,97 \pm 11,99$ & $\mathrm{NQ}$ & $\mathrm{NQ}$ \\
Novozym 188 & $\mathrm{NQ}$ & $352,62 \pm 10,71$ & $\mathrm{NQ}$ \\
NS22074 & $29,84 \pm 0,35$ & $\mathrm{NQ}$ & $\mathrm{NQ}$ \\
NS50010 & $\mathrm{NQ}$ & $1236,11 \pm 60,98$ & $\mathrm{NQ}$ \\
NS22036 & $\mathrm{NQ}$ & $\mathrm{NQ}$ & $599,64 \pm 7,77$ \\
NS50012 & $2,07 \pm 0,88$ & $1121,6 \pm 96,04$ & $160,87 \pm 4,15$ \\
Accellerase 1500 & $52,69 \pm 3,17$ & $88,16 \pm 2,68$ & $\mathrm{NQ}$ \\
\hline
\end{tabular}

NQ - não quantificado

Na Tabela 3 estão apresentados os resultados da composição do bagaço de cana-deaçúcar na forma in natura e a fração sólida do bagaço pré-tratado.

Tabela 3 - Composição do bagaço de cana-de-açúcar in natura e fração sólida do pré-tratado com $(5,0 \pm 1,5 \%)$ de umidade.

\begin{tabular}{ccc}
\hline Componentes (\%) & In natura & Pré-tratado \\
\hline Celulose & $38,59 \pm 3,45$ & $64,89 \pm 1,57$
\end{tabular}




$\begin{array}{ccc}\text { Hemicelulose } & 27,89 \pm 2,68 & 9,61 \pm 0,94 \\ \text { Lignina total } & 17,79 \pm 0,62 & 7,85 \pm 0,24 \\ \text { Extrativos em solvente } & 1,61 \pm 0,16 & 0,71 \pm 0,04 \\ \text { orgânico } & & \\ \text { Extrativos em água } & 1,11 \pm 1,23 & 11,23 \pm 0,53 \\ \text { quente } & 8,80 \pm 0,02 & 5,75 \pm 0,14 \\ \text { Cinzas } & \end{array}$

Conforme Tabela 3, o teor de celulose teve um aumento de 68,33\% para o bagaço prétratado e, para hemicelulose, observou-se uma redução de 63,15\%. A redução de hemicelulose no bagaço submetido ao pré-tratamento se deve ao fato de se usar substâncias ácidas como catalisador na primeira etapa do pré-tratamento (Schell et al., 2003). O teor de hemicelulose que fica no hidrolisado líquido pode ser utilizado para diferentes estudos como produção de etanol a partir de xilose, produção de xilitol, dentre outros, mas não foi o caso deste trabalho.

Os valores de lignina reduziram em 55,87\% para o bagaço pré-tratado. Esta redução é importante para aumentar o contato das enzimas com a celulose e diminuir a adsorção da lignina com as enzimas, tal efeito foi obtido devido ao fato de que o pré-tratamento utilizado ser em meio alcalino na última etapa (Chang e Holtzapple, 2000). A lignina hidrolisada também pode ser utilizada como matéria-prima para outros processos dentro de uma biorrefinaria, entretanto, não foi o foco deste trabalho.

Canilha et al., (2011) ao aplicar a metodologia de planejamento experimental no processo de pré-tratamento utilizando ácido sulfúrico encontrou a melhor condição de $150^{\circ} \mathrm{C}$ por 30 minutos e $2,5 \%$ de ácido sulfúrico, sendo os resultados alcançados de $59,3 \%$ de celulose, $3,7 \%$ de hemicelulose e $33,8 \%$ de lignina, partindo de um bagaço de cana com $45 \%$ de celulose, $25,8 \%$ de hemicelulose e $19,1 \%$ de lignina. Os resultados de redução de hemicelulose são compatíveis com este trabalho. Já para a lignina, a redução obtida no trabalho foi devido ao fato de se aplicar uma segunda etapa no pré-tratamento sendo esta alcalina.

Com relação aos extrativos em solvente orgânico, observou-se uma redução no bagaço pré-tratado. Já os valores de extrativos em água quente aumentaram. Estes valores podem ter aumentado devido ao pré-tratamento deixar resíduos dos compostos monoméricos solubilizados de forma ainda adsorvida à matriz lignocelulósica, mas que é removida por água a $100^{\circ} \mathrm{C}$. A quantidade de cinzas também foi reduzida. Esta redução, tanto no teor de cinzas quanto de extrativos em solvente orgânico estão em concordância com a literatura (Alvira et al., 2009).

Nas Figuras 1 e 2 estão apresentados os resultados de hidrólise enzimática realizadas em bagaço de cana pre-tratado para e seleção da mistura enzimática, bem como, teste com enzimas xilanases, hemicelulases e a adição de TWEEN 80 ao processo. 


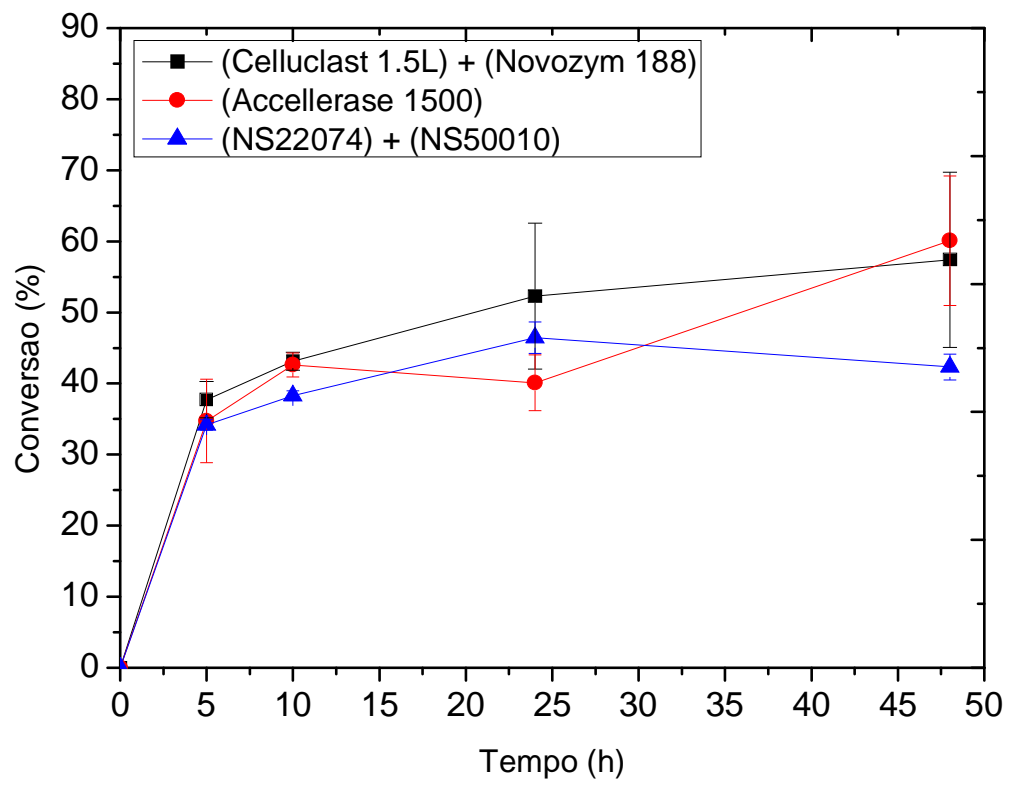

Figura 1 - Ensaios enzimáticos; primeiro $(\boldsymbol{\bullet})$, segundo $(\bullet)$ e terceiro $(\boldsymbol{\Delta})$ estudo para seleção da melhor mistura enzimática.

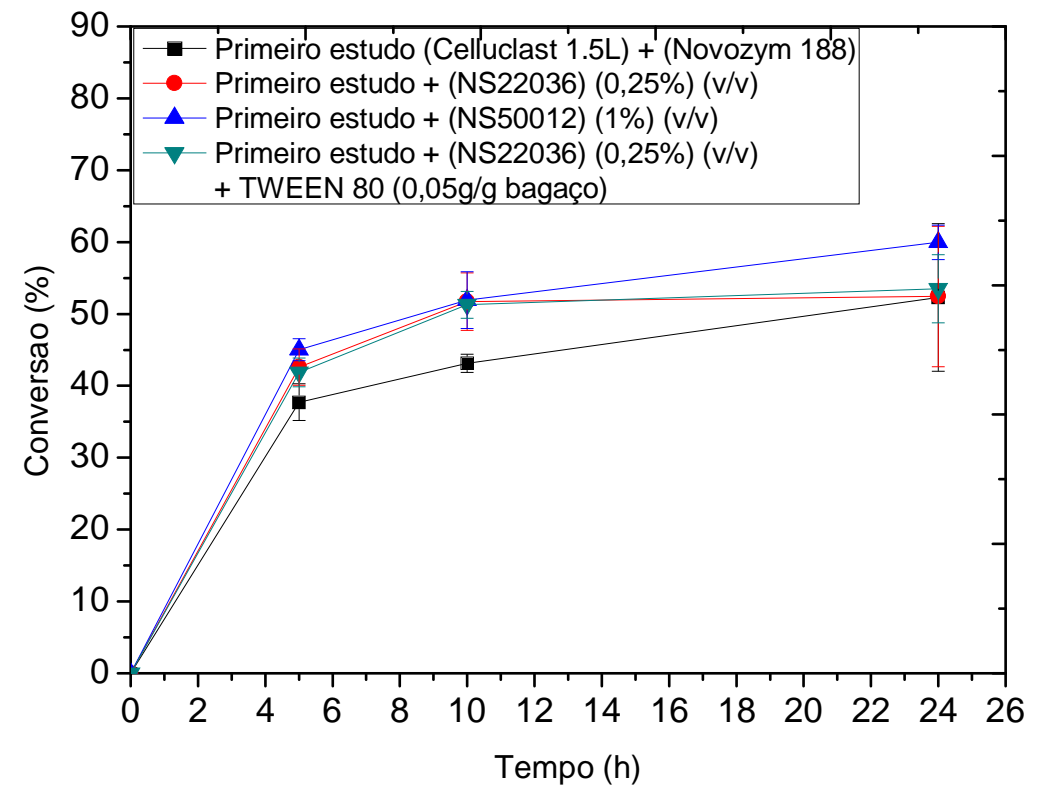

Figura 2 - Ensaios enzimáticos; primeiro estudo (•), para fins de comparação, quarto $(\bullet)$, quinto $(\boldsymbol{\Delta})$ e sexto estudo $(\boldsymbol{\nabla})$. 
De acordo com as análises dos resultados (Figura 1), foi possível observar que não existe diferença entre a glicose produzida nas hidrólises enzimáticas nos primeiro, segundo e terceiro estudo nos tempos e condições estudadas (dados não demonstrados), com exceção do terceiro estudo que foi diferente estatisticamente do segundo estudo no tempo de 48 horas (p $=0,029)$. Este último resultado, muito provavelmente, foi consequência de erros experimentais.

Pelas análises dos resultados a partir da Figura 2, foi visto que o teor de celulose digerida aumentou no quarto, quinto e sexto estudo no tempo de 10 horas, quando comparado ao primeiro estudo $(p=0,024)(p=0,021)$ e $(p=0,003)$, respectivamente. Também foi verificado que, no tempo de 5 e 10 horas, não há diferença entre os quarto, quinto e sexto estudo (dados não demonstrados). Para os tempos de 24 horas, não há diferença estatística no teor de celulose digerida para o primeiro, quarto, quinto e sexto estudo, $(\mathrm{p}=0,47),(\mathrm{p}=$ 0,052), $(\mathrm{p}=0,17)$, respectivamente. Portanto, a adição das enzimas xilanases só seria útil se o tempo de hidrólise fosse de 10h. Já o uso do TWEEN 80 não foi viável nestas condições.

Para todos os ensaios foi possível observar valores de conversão entre 50 a $60 \%$, cabe aqui, se fazer uma análise de custo quanto os tipos de enzimas e reagentes aplicados nos estudos para se determinar o ensaio com maior lucro. Resultados de conversão próximos aos encontrados neste trabalho podem ser visto na literatura (Borjesson et al., 2007 e Maeda et al,. 2011).

Acredita-se que exista inibição do processo aqui estudado devido aos produtos formados, celobiose e glicose, e que uma integração entre o processo de hidrólise enzimática e um processo de fermentação alcóolica pode favorecer o deslocamento da reação para a direita e, assim, aumentando os valores de conversão, bem como produzindo produtos de valor agregado como o etanol a partir de resíduos da agroindústria.

\section{CONCLUSÃO}

De acordo com os resultados obtidos neste trabalho é possível verificar que com o prétratamento realizado no bagaço de cana proporciona um aumento de $68 \%$ de celulose no bagaço pré-tratado e, após as hidrólises enzimáticas, pode-se obter até $60 \%$ de conversão de celulose em glicose.

O uso do TWEEN 80 não foi viável nestas condições de processo aqui aplicadas, entretanto, outros estudo devem ser realizados com outras concentrações deste reagente.

Uma avaliação econômica mais detalhada deve ser feita para a escolha do melhor processo aqui apresentado em termos de usos das enzimas xilanases e hemicelulases adicionadas.

Novos pré-tratamentos podem ser testados para estudar sua influência na digestibilidade da celulose no bagaço de cana-de-açúcar, também se podem realizar planejamentos experimentais para se determinar quantidades das celulases e $\beta$-glicosidases iniciais no processo visando redução de custos.

Um estudo de hidrólise enzimática deveria ser realizado em biorreator para se melhorar o processo de transferência de massa bem como se realizar um estudo de ampliação de escala. 


\section{REFERÊNCIAS}

ALVIRA, P, TOMAS-PEJO, E.; BALlESTEROS, M.; NEGRO.; M. J. Pretreatment technologies for an efficient bioethanol production process based on enzymatic hydrolysis: A review. Bioresour Technol, v. 101, p. 4851-4861, 2009.

BORJESSON, J.; PETERSON, R.; TJERNELD, F. Enhanced enzymatic conversion of softwood lignocellulose by poly(ethylene glycol) addition. Enzyme Microb Technol, v. 40, p. 754-762, 2007.

CANILHA, L, SANTOS, V. T. O.; ROCHA, G. J. M.; SIlVA, J. B. A.; GIULIETTI, M.; SILVA, S. S.; FELIPE, M. G. A.; FERRAZ, A.; MILAGRES, A. M. F.; CARVALHO, W. A study on the pretreatment of a sugarcane bagsse sample with dilute sulfuric acid. J Ind Microbiol Biotechnol, v. 38, p. 1467-1475, 2011.

CHANG, V.; HOLTZAPPLE, M. Fundamental factors affecting biomass enzymatic reactivity. Appl Biochem Biotechnol, v. 84-86, p. 5-37, 2000.

GALBE, M.; ZACCHI, G. A review of the production of ethanol from softwood. Appl Microbiol Biotechnol, v. 59 , p. 618-628, 2002.

GHOSE, T. K. Measurement of cellulose activities. Pure Appl Chem. v. 59, p. 257-268, 1987.

GOUVEIA, E. R.; NASCIMENTO, R. T.; SOUTO-MAIOR, A. M.; ROCHA, G. J. M. Validação de metodologia para a caracterização química de bagaço de cana-de-açúcar. Quim Nova, v. 32, p. 1500-1503, 2009.

GUO, G. L.; HSU, D. C.; CHEN, W. H.; CHEN, W.H.; HWANG, W. S. Characterization of enzymatic saccharification for acid-pretreated lignocellulosic materials with different lignin composition. Enzyme Microb Technol, v. 45, p. 80-87, 2009.

HAN Y.; CHEN H. Characterization of $\beta$-glucosidase from corn stover and its application in simultaneous saccharification and fermentation. Bioresour Technol, v. 99, p. 6081-6087, 2008.

LYND, L.; WEIMER, P.; ZYL, W.; PRETORIUS, A. Microbial cellulose utilization: fundamentals and biotechnology. Microbiol Mol Bio Rev, v. 66, p. 506-577, 2002.

MAEDA, R. N.; SERPA, V. I.; ROCHA, V. A. L.; MESQUITA, R. A. A.; ANNA, L. M. M. S.; CASTRO, A. M.; DRIEMEIER, C. E.; PEREIRA, N. Jr.; POLIKARPOV, I. Enzymatic hydrolysis of pretreated sugar cane bagasse using Penicillium funiculosum and Trichoderma harzianum celulases. Process Biochem. v. 46, p. 11961201,2011

SELIG, M.; WEISS, N.; JI, Y. Enzymatic Saccharification of Lignocellulosic Biomass. Laboratory Analytical Procedure (LAP), Technical Report, NREL, 2008. p. 8.

SCHELL, D. J.; FARMER, J.; NEWMAN, M.; MCMILLAN, J. D. Dilutesulfuric acid pretreatment of corn stover in pilot-scale reactor - investigation of yields, kinetics, and enzymatic digestibilities of solids. Appl Biochem Biotechnol, v. 105, p. 69-85, 2003.

SLUITER, A.; RUIZ, R.; SCARLATA, C.; SLUITER, L.; TEMPLETON, D. Determination of Extractives in Biomass. Laboratory Analytical Procedure (LAP), Technical Report, NREL, 2008. p. 12.

VASQUEZ, M. P.; SILVA, J. N. C.; SOUZA, M. B. Jr.; PEREIRA, N. Jr. Enzymatic hydrolysis optimization to ethanol production by simultaneous saccharification and fermentation. Appl Biochem Biotechnol, v. 136-140, p. 141-154, 2007.

ZHOU, J.; WANG, Y. H.; CHU, J.; LUO, L. Z.; ZHUANG, Y. P.; ZHANG, S. L. Optimization of cellulase mixture for efficient hydrolysis of steam-exploded corn stover by statistically designed experiments. Bioresour Technol, v. 100, p. 819-825, 2009. 